\title{
End-to-End Entity Resolution and Question Answering Using Differentiable Knowledge Graphs
}

\author{
Armin Oliya Amir Saffari Priyanka Sen Tom Ayoola \\ Amazon Alexa AI \\ Cambridge, UK \\ \{aooliya, amsafari, sepriyan, tayoola\} @amazon.com
}

\begin{abstract}
Recently, end-to-end (E2E) trained models for question answering over knowledge graphs (KGQA) have delivered promising results using only a weakly supervised dataset. However, these models are trained and evaluated in a setting where hand-annotated question entities are supplied to the model, leaving the important and non-trivial task of entity resolution (ER) outside the scope of E2E learning. In this work, we extend the boundaries of E2E learning for KGQA to include the training of an ER component. Our model only needs the question text and the answer entities to train, and delivers a stand-alone QA model that does not require an additional ER component to be supplied during runtime. Our approach is fully differentiable, thanks to its reliance on a recent method for building differentiable KGs (Cohen et al., 2020). We evaluate our E2E trained model on two public datasets and show that it comes close to baseline models that use handannotated entities.
\end{abstract}

\section{Introduction}

The conventional approach for Question Answering using a Knowledge Graph (KGQA) involves a set of loosely connected components; notably, an entity resolution component identifies entities mentioned in the question, and a semantic parsing component produces a structured representation of the question. The programs resulting from combining these components can be executed on a knowledge graph (KG) engine to retrieve the answers.

While this approach can be effective, collecting training datasets for individual components can be challenging (Dahl et al., 1994; Finegan-Dollak et al., 2018). For example, supervised semantic parsing requires training data pairing naturallanguage questions with structured queries, which is difficult to obtain. This has motivated many efforts in weakly supervised training (Chakraborty et al., 2021). Following recent breakthroughs in

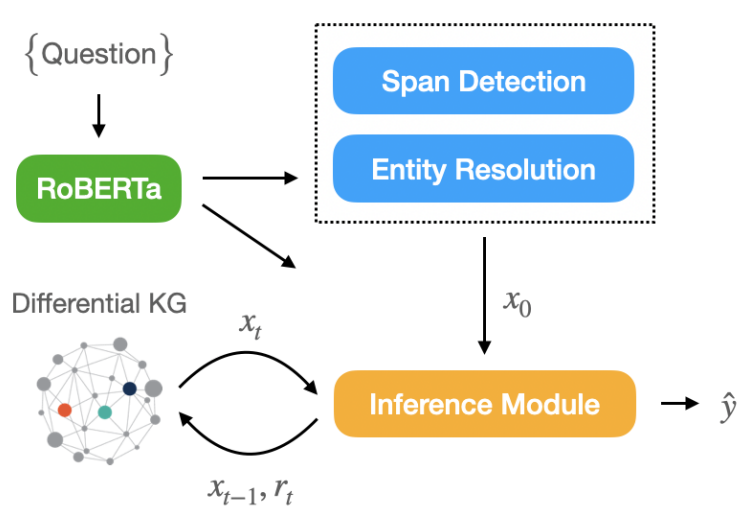

Figure 1: High-level architecture of the end-to-end model. One forward pass of RoBERTa extracts contextual embeddings for all components. Span Detection and Entity Resolution happen jointly to derive seed entities vector $\boldsymbol{x}_{\mathbf{0}}$. The inference module performs multihop reasoning to reach answer entities vector $\hat{\boldsymbol{y}}$

machine translation (Bahdanau et al., 2015), a new goal is to directly optimize the entire chain of components end-to-end, without the need for intermediate annotations.

However, entity resolution (ER) is by and large a neglected component of E2E learning, and existing weakly supervised solutions mostly assume question entities are either given or extracted by an external system. In practice, there's a scarcity of quality training data for ER on questions, and poor entity extraction by out-of-domain models affects the overall performance of a KGQA system (Singh et al., 2020; Han et al., 2020).

In this work, we present an end-to-end model for KGQA that learns to jointly perform entity resolution and inference. Our work leverages the differentiable KG proposed in Cohen et al. (2020), which allows all the components of our model to be trained using a dataset of only questions and answers. This eliminates the need for labelled ER data for questions and allows our model to run independently, without relying on external components. Furthermore, the tight integration of ER into our 
solution allows uncertainties about entities to be directly reflected in our confidence in answers.

\section{Related Work}

Traditional approaches to KGQA rely on semantic parsing (SP) to translate natural language into a logical form. Weakly supervised SP is a wellstudied topic with increasing interest in applying Reinforcement Learning (RL) (Hua et al., 2020; Agarwal et al., 2019). ER is rarely considered in the scope of surveyed solutions, and if it is, it's treated as an independent component and not included in the weak supervision scope (Ansari et al., 2019). In general, RL algorithms for QA are hard to tune and have large variances in their results. The exploration-exploitation issue also lets models settle on high-reward but spurious logical forms, leading to poor generalization (Chakraborty et al., 2021). Including ER with a discrete output space as part of an E2E RL pipeline will further add to the challenges that RL-based solutions face.

Another line of work in KGQA uses embedding techniques to implicitly infer answers from knowledge graphs without explicit queries (Saxena et al., 2020; Sun et al., 2019). While these embeddingbased approaches perform well, they are memory intensive and difficult to scale to large knowledge graphs. In addition, when new entities are added to the KG, they need to be retrained to learn updated embeddings. The differentiable KG we use in this work can incorporate new entities without affecting trained models, and can scale to billions of entities via horizontal scaling (Cohen et al., 2020).

The few relevant works on entity resolution for questions utilize complex models with many interworking modules (e.g. Sorokin and Gurevych 2018; Tan et al. 2017). ELQ (Li et al., 2020) is a more recent effort and simplifies the process by relying on a bi-encoder to jointly perform span detection and ER in a multi-task setup. However, these solutions rely on direct supervision. Our proposed method eliminates the need for labelled data for ER. In fact, the weakly supervised ER model presented here could be detached and used as a standalone ER module after training.

\section{E2E Model}

\subsection{Differentiable Knowledge Graph}

A traditional knowledge graph (KG) stores facts as triples and uses a symbolic query engine to extract answers. A differentiable KG stores facts in tensors and makes query execution over facts differentiable.

We use the approach presented in ReifiedKB (Cohen et al., 2020) to create a scalable and differentiable knowledge graph supporting multi-hop relation following programs. We provide an overview here but full details can be found in the original paper. Assume the set of all triples in a knowledge graph $\mathcal{T}=\left\{t_{i}\right\}_{i=1}^{N_{T}}, \quad t_{i}=\left(s_{s_{i}}, p_{p_{i}}, o_{o_{i}}\right)$ are represented by the following sparse matrices:

$$
\begin{array}{ll}
M_{s} \in\{0,1\}^{N_{T} \times N_{E}}, & M_{s}(i, j)=\mathbb{I}\left(e_{j}=s_{s_{i}}\right) \\
M_{o} \in\{0,1\}^{N_{T} \times N_{E}}, & M_{o}(i, j)=\mathbb{I}\left(e_{j}=o_{o_{i}}\right) \\
M_{p} \in\{0,1\}^{N_{T} \times N_{R}}, & M_{p}(i, j)=\mathbb{I}\left(p_{j}=p_{p_{i}}\right),
\end{array}
$$

where $M_{s}, M_{o}$, and $M_{p}$ are denoted as subject, object, and relation index matrices. $N_{T}, N_{E}$, and $N_{R}$ are the number of triples, entities, and relations, respectively.

Given an entities vector $\mathbf{x}_{t-1} \in \mathbb{R}^{N_{E}}$ at $t-1$-th hop, the entities vector $\mathbf{x}_{t}$ resulting from following a relation vector $\mathbf{r}_{t} \in \mathbb{R}^{N_{R}}$ can be computed by:

$$
\mathbf{x}_{t}=\text { follow }\left(\mathbf{x}_{t-1}, \mathbf{r}_{t}\right)=M_{o}^{T}\left(M_{s} \mathbf{x}_{t-1} \odot M_{p} \mathbf{r}_{t}\right),
$$

where $\odot$ is the element-wise multiplication.

\subsection{Multi-hop Inference}

Given an input question $q=q_{1} \cdots q_{n}$ of length $n$, we first use the pretrained language model RoBERTa (Liu et al., 2019) to extract contextual embeddings for each token:

$$
\left[\mathbf{h}_{\mathbf{q}}, \boldsymbol{q}_{\mathbf{1}} \cdots \boldsymbol{q}_{\boldsymbol{n}}\right]^{T}=L M\left(C L S, q_{1} \cdots q_{n}\right)
$$

where $\boldsymbol{h}_{\boldsymbol{q}} \in \mathbb{R}^{D}$ corresponds to the $C L S$ token and is used as the question embedding. We compute the relation vector for the $t$-th hop using a hierarchical decoder and subsequent entities vector by following that vector as:

$$
\begin{aligned}
& \mathbf{r}_{t}=\operatorname{softmax}\left(\mathbf{W}_{\mathbf{t}}^{\inf }\left[\mathbf{h}_{q}\left|\mathbf{r}_{t-1}\right| \cdots \mid \mathbf{r}_{1}\right]^{T}\right), \\
& \mathbf{x}_{t}=\operatorname{follow}\left(\mathbf{x}_{t-1}, \mathbf{r}_{t}\right) .
\end{aligned}
$$

Since the follow operation (Equation 1) can be chained infinitely, we set a maximum number of hops and use an attention mechanism to combine answer entities across all hops. We compute attention across all hops by:

$$
\begin{aligned}
\mathbf{c}_{\mathbf{t}} & =\mathbf{W}_{\mathbf{t}}^{\mathbf{a t t}}\left[\mathbf{h}_{q}\left|\mathbf{r}_{t-1}\right| \cdots \mid \mathbf{r}_{1}\right]^{T}, \\
\mathbf{a} & =\operatorname{softmax}\left(\left[\mathbf{c}_{\mathbf{1}}, \cdots, \mathbf{c}_{\mathbf{T}_{\max }}\right]\right),
\end{aligned}
$$


where $T_{\max }$ is the predefined maximum number of hops. The final answer entities vector will be:

$$
\hat{\mathbf{y}}=\sum_{t=1}^{T_{\max }} a_{t} \mathbf{x}_{t}
$$

Compared to ReifiedKB, our decoder uses RoBERTa for embedding questions, simplifies the stopping mechanism, and allows returning more than one answer entity.

\subsection{Entity Resolution}

We approach Entity Resolution by estimating the likelihood of all the plausible spans in the question and then selecting the most likely candidate entity for each span.

Given an input question $q=q_{1} \cdots q_{n}$ of length $n$, the likelihood of each span $[i, j]$ in the question ( $i$-th to $j$-th tokens of $q$ ) is calculated as:

$$
\begin{aligned}
\boldsymbol{q}_{\boldsymbol{i j}} & =\frac{\sum_{i}^{j} \boldsymbol{q}_{\boldsymbol{k}}}{(j-i+1)} \in \mathbb{R}^{D}, \\
s_{i j} & =\frac{\exp \left(\boldsymbol{q}_{\boldsymbol{i j}} \boldsymbol{w}_{\boldsymbol{s}}\right)}{\sum_{\forall m, n} \exp \left(\boldsymbol{q}_{\boldsymbol{m} \boldsymbol{n}} \boldsymbol{w}_{\boldsymbol{s}}\right)},
\end{aligned}
$$

where $\boldsymbol{w}_{\boldsymbol{s}} \in \mathbb{R}^{D \times 1}$ is a learnable matrix and $\boldsymbol{q}_{\boldsymbol{k}}$ are contextual token embeddings from Equation 2.

For a given span, candidate entities that could be referred to by that span are extracted by exact search against a lookup table, built using titles and aliases of entities in the KG. Candidate generation can further be improved by considering other approximate or fuzzy search methods, but we leave this as future work.

If there are overlapping candidates between two spans, they are assigned to the longer one. For example, consider the question "what position does carlos gomez play?". If candidates of the three spans "carlos", "gomez", and "carlos gomez" all contain Q2747238 (the Wikidata entity ID referring to Carlos Gomez, the Dominican baseball player), the entity ID will be assigned to the longest span only ("carlos gomez"). This is to avoid having duplicate entities across spans and comes from the intuition that longer spans are more specific and should be preferred. We have not seen errors arising from this preprocessing step.

Assume $c_{i j}^{k}$ is the $k$-th candidate for span $[i, j]$. We embed each candidate entity by learning a dense representation of its $\mathrm{KG}$ neighbourhood:

$$
\begin{aligned}
\mathcal{F}_{i j}^{k} & =\left\{f_{\text {em }}([p \mid o]) \forall\left(c_{i j}^{k}, p, o\right) \in \mathcal{G}\right\}, \\
\mathbf{z}_{\mathbf{i j}}^{\mathbf{k}} & =\frac{\sum_{\boldsymbol{v} \in \mathcal{F}_{i j}^{k}} \boldsymbol{v}}{\left|\mathcal{F}_{i j}^{k}\right|} \in \mathbb{R}^{D},
\end{aligned}
$$

where $\mathcal{G}$ is the knowledge graph, $[p \mid o]$ is the string concatenation of $p$ and $o$, and $f_{e m}$ is an embedding function that maps a string to a dense vector.

For example, assume $Q 2747238$ is the candidate entity we want to embed. It is on the left-hand side of two triples in the knowledge graph: (Q2747238, instance-of, human) and (Q2747238, occupation, baseball player).

We first create the two strings "instanceof: human" and "occupation: baseball player" and pass them to $f_{e m}$ to embed. These strings are treated as features of 22747238 , and we are able to learn embeddings effectively since they are also used for other humans and baseball players. Finally, we take the average of these two feature embeddings to get to the entity embedding for Q2747238. These operations are implemented using torch.nn.EmbeddingBag in PyTorch with random initialization. Our approach is not limited by knowledge graph features or this specific embedding approach; for instance, a RoBERTa encoding of entity descriptions could be used as an alternative. We leave experiments with other entity representations as future work.

Given the embedding, the likelihood of a candidate entity is estimated by considering the span likelihood and the likelihood of other candidates in that span:

$$
e_{i j}^{k}=s_{i j} \frac{\exp \left(\left[\mathbf{z}_{\mathbf{i j}}^{\mathbf{k}} \cdot \boldsymbol{q}_{\boldsymbol{i} \boldsymbol{j}}\right]\right)}{\sum_{\forall l} \exp \left(\left[\mathbf{z}_{\mathbf{i j}}^{\mathbf{l}} \cdot \boldsymbol{q}_{\boldsymbol{i j}}\right]\right)} .
$$

To get to the final entity vector, we re-score candidate entities across all spans:

$$
\begin{aligned}
x_{i j}^{k} & =\frac{\exp \left(e_{i j}^{k}\right)}{\sum_{\forall u, v, w} \exp \left(e_{u v}^{w}\right)}, \\
\mathbf{x}_{\mathbf{0}} & =x_{i j}^{k} \mapsto \overrightarrow{0} \in \mathbb{R}^{N_{E}},
\end{aligned}
$$

where $\mapsto$ maps each candidate entity likelihood to its corresponding index in the zero vector $\overrightarrow{0}$. The resulting $\mathbf{x}_{0}$ vector is used in Equation 4 and captures uncertainties about entity resolution. It is different from Cohen et al. 2020 where $\mathbf{x}_{0}$ is assumed to be given with $\{0,1\}$ as the only possible values. 


\subsection{Training}

We train the model using the binary cross-entropy loss function:

$\mathcal{L}(\mathbf{y}, \hat{\mathbf{y}})=\frac{1}{N_{E}} \sum_{i=1}^{N_{E}} y_{i} \log \hat{y}_{i}+\left(1-y_{i}\right) \log \left(1-\hat{y}_{i}\right)$,

where $\mathbf{y} \in \mathbb{R}^{N_{E}}$ is a $k$-hot label vector. While ReifiedKB uses cross-entropy loss, we instead use a multi-label loss function across all entities. This is because the output space in a majority of cases contains multiple entities, so cross-entropy loss is inadequate. During training, the entity resolution and inference modules are trained jointly and uncertainties about each module are propagated to final answer entities vector $\hat{\mathbf{y}}$.

\section{Experiments}

We call our model Rigel and evaluate 3 versions at different E2E learning boundaries. The baseline model, Rigel-Baseline, is given gold entities and no entity resolution is involved, demonstrating the performance of the inference module alone. Rigel$E R$ is given the gold spans, but still has to learn to disambiguate between candidate entities for that span. Finally, in Rigel-E2E, we provide the question text only, requiring the model to attend to the right span and disambiguate between candidates for each span.

\subsection{Datasets}

We evaluate our models on two open-domain Question Answering datasets: SimpleQuestions (Bordes et al., 2015) and WebQSP (Yih et al., 2016). Both datasets were constructed based on the outdated FreeBase. Therefore, to generate better candidates and entity representation, we chose to use a subset of these datasets that are answerable by Wikidata (Diefenbach et al., 2017). This is different from other baselines we compare against, which do not include an ER component. For WebQSP, this leads to 2349 train, $261 \mathrm{dev}$, and 1375 test set samples. For SimpleQuestions the number of samples are 19471 train, $2818 \mathrm{dev}$, and 5620 test.

Questions in SimpleQuestions and WebQSP can be answered in 1 and 2 hops respectively, so we set the maximum number of hops $T_{\max }$ in Equation 7 accordingly. For each dataset, we also limit Wikidata to a subset that is $T_{\max }$-hop reachable from any of the candidates $c_{i j}^{k}$ in Equation 10. This results in a subgraph with 3.7 million triples, 1.0 million entities, and 1,158 relations for SimpleQuestions; and 4.9 million triples, 1.1 million entities, and 1,230 relations for WebQSP.

\subsection{Results}

Results of our experiments are shown in Table 1. We don't directly compare to other related work since their performance is reported with access to gold-entities and their quality when building a practical QA system with an external ER is unknown.

Compared to Rigel-Baseline, there is approximately a $3 \%$ drop in performance when gold question entities are not provided to the model (Rigel$E R$ ). We realized this is mainly due to cases where it is not possible to distinguish between all possible candidate entities based on the question alone. This is consistent with earlier studies that conclude $15-17 \%$ of questions in these datasets cannot be answered due to context ambiguity (Han et al., 2020; Petrochuk and Zettlemoyer, 2018). For example, in the question "What position does carlos gomez play?" ("carlos gomez" given as correct span), Rigel-ER learns to give higher likelihood to athletes compared to art performers; but since the question does not include discriminative information such as sport or team name, all athletes called "Carlos Gomez" will receive very similar likelihood scores.

There is a further drop in performance when we go from Rigel-ER to Rigel-E2E, which performs full E2E learning. This time, the errors can be explained by the fact that different spans produce candidates with overlapping entity types, leaving the model with little signal to prefer one span over another.

For example, given the question "who directed the film gone with the wind?", Rigel-ER is given the correct span "gone with the wind" and just needs to disambiguate between $\mathrm{Q} 2875$ (the Wikidata entity ID for the American film "Gone with the Wind") and the other candidates stemming from that span. Rigel-E2E will additionally need to learn to maximize the span score (Equation 9) for "gone with the wind" compared to other spans in the question, such as "the film", "the wind", and "wind", which are all film titles as well. This is a difficult task since all these spans produce film entities, and relying on the loss from following the director relation is not enough to effectively disambiguate between them.

We are working on a few solutions to allevi- 
ate this span ambiguity issue with Rigel-E2E. The main question is, what should we do when span scores are diffused and not spiked? This, for example, happens in the above question and the 4 spans: "gone with the wind" , "the film", "the wind", and "wind". A simple post-processing step to merge overlapping spans seems to be quite effective. In the example above, "the wind" and "wind" fall under "gone with the wind", and given that their scores are similar we can decide to assign all child span scores to their parent. Diversity or entropy of candidates produced by a certain span also seems to be helpful in pruning bad spans. In the above question, candidate entities from the span "wind" include movies, companies, music bands, and even a satellite, among others. On the other hand, candidate entities for "gone with the wind" are mostly works of art, suggesting that it may be a better choice. We are looking into using this information as part of training, as well as post-processing.

While we don't directly compare, the gap between our results and other related work is partly due to the inference mechanism used. At this time, ReifiedKB only supports a relation following operation (Equation 1), while, for instance, EmQL (Sun et al., 2020) additionally supports set intersection, union, and difference. These additional operations allow answering more complex questions present in the WebQSP dataset. We are working on adding support for intersection, union, count, min, and max operations to our model as future work.

We'd like to emphasize that although including the ER component adversely affects the results, extracting question entities is a necessity for real world applications, and alternatives with off-theshelf models do perform worse. Hence, we believe our approach is more practical, especially given the lack of training data for ER on questions.

\section{Conclusion}

In this work, we proposed a solution for KGQA that jointly learns to perform entity resolution (ER) and multi-hop inference. Our model extends the boundaries for end-to-end learning and is weakly supervised using pairs of only questions and answers. This eliminates the need for external components and expensive domain-specific labelled data for ER. We further demonstrate the feasibility of this approach on two open-domain QA datasets.

\begin{tabular}{lcc}
\hline Model & WQSP & SIQ \\
\hline KVMem (Miller et al., 2016) & 46.7 & - \\
ReifiedKB (Cohen et al., 2020) & 52.7 & - \\
EmQL (Sun et al., 2020) & 75.5 & - \\
MemNN (Bordes et al., 2015) & - & 61.6 \\
KBQA-Adapter (Wu et al., 2019) & - & 72.0 \\
\hline Rigel-Baseline & 52.4 & 73.4 \\
Rigel-ER & 48.2 & 70.1 \\
Rigel-E2E & 45.0 & 68.2 \\
\hline
\end{tabular}

Table 1: Comparison of Hits@1 results on WebQSP (WQSP) and Accuracy on SimpleQuestions (SIQ)

\section{References}

Rishabh Agarwal, Chen Liang, Dale Schuurmans, and Mohammad Norouzi. 2019. Learning to generalize from sparse and underspecified rewards. In Proceedings of the 36th International Conference on Machine Learning, volume 97 of Proceedings of Machine Learning Research, pages 130-140. PMLR.

Ghulam Ahmed Ansari, Amrita Saha, Vishwajeet Kumar, Mohan Bhambhani, Karthik Sankaranarayanan, and Soumen Chakrabarti. 2019. Neural program induction for kbqa without gold programs or query annotations. In Proceedings of the Twenty-Eighth International Joint Conference on Artificial Intelligence, IJCAI-19, pages 4890-4896. International Joint Conferences on Artificial Intelligence Organization.

Dzmitry Bahdanau, Kyunghyun Cho, and Yoshua Bengio. 2015. Neural machine translation by jointly learning to align and translate. In 3rd International Conference on Learning Representations, ICLR 2015, San Diego, CA, USA, May 7-9, 2015, Conference Track Proceedings.

Antoine Bordes, Nicolas Usunier, Sumit Chopra, and Jason Weston. 2015. Large-scale simple question answering with memory networks. CoRR, abs/1506.02075.

Nilesh Chakraborty, Denis Lukovnikov, Gaurav Maheshwari, Priyansh Trivedi, Jens Lehmann, and Asja Fischer. 2021. Introduction to neural networkbased question answering over knowledge graphs. WIREs Data Mining and Knowledge Discovery, 11(3):e1389.

William W. Cohen, Haitian Sun, R. Alex Hofer, and Matthew Siegler. 2020. Scalable neural methods for reasoning with a symbolic knowledge base. In 8th International Conference on Learning Representations, ICLR 2020, Addis Ababa, Ethiopia, April 26-30, 2020. OpenReview.net.

Deborah A. Dahl, Madeleine Bates, Michael Brown, William Fisher, Kate Hunicke-Smith, David Pallett, 
Christine Pao, Alexander Rudnicky, and Elizabeth Shriberg. 1994. Expanding the scope of the atis task: The atis-3 corpus. In Proceedings of the Workshop on Human Language Technology, HLT '94, page 43-48, USA. Association for Computational Linguistics.

Dennis Diefenbach, Thomas Pellissier Tanon, Kamal Deep Singh, and Pierre Maret. 2017. Question answering benchmarks for wikidata. In Proceedings of the ISWC 2017 Posters \& Demonstrations and Industry Tracks co-located with 16th International Semantic Web Conference (ISWC 2017), Vienna, Austria, October 23rd - to - 25th, 2017.

Catherine Finegan-Dollak, Jonathan K. Kummerfeld, Li Zhang, Karthik Ramanathan, Sesh Sadasivam, Rui Zhang, and Dragomir Radev. 2018. Improving text-to-SQL evaluation methodology. In Proceedings of the 56th Annual Meeting of the Association for Computational Linguistics (Volume 1: Long Papers), pages 351-360, Melbourne, Australia. Association for Computational Linguistics.

Namgi Han, Goran Topic, Hiroshi Noji, Hiroya Takamura, and Yusuke Miyao. 2020. An empirical analysis of existing systems and datasets toward general simple question answering. In Proceedings of the 28th International Conference on Computational Linguistics, pages 5321-5334, Barcelona, Spain (Online). International Committee on Computational Linguistics.

Yuncheng Hua, Yuan-Fang Li, Guilin Qi, Wei Wu, Jingyao Zhang, and Daiqing Qi. 2020. Less is more: Data-efficient complex question answering over knowledge bases. Journal of Web Semantics, 65:100612.

Belinda Z. Li, Sewon Min, Srinivasan Iyer, Yashar Mehdad, and Wen-tau Yih. 2020. Efficient one-pass end-to-end entity linking for questions. In Proceedings of the 2020 Conference on Empirical Methods in Natural Language Processing (EMNLP), pages 6433-6441, Online. Association for Computational Linguistics.

Yinhan Liu, Myle Ott, Naman Goyal, Jingfei Du, Mandar Joshi, Danqi Chen, Omer Levy, Mike Lewis, Luke S. Zettlemoyer, and Veselin Stoyanov. 2019. Roberta: A robustly optimized bert pretraining approach. ArXiv, abs/1907.11692.

Alexander Miller, Adam Fisch, Jesse Dodge, AmirHossein Karimi, Antoine Bordes, and Jason Weston. 2016. Key-value memory networks for directly reading documents. In Proceedings of the 2016 Conference on Empirical Methods in Natural Language Processing, pages 1400-1409, Austin, Texas. Association for Computational Linguistics.

Michael Petrochuk and Luke Zettlemoyer. 2018. SimpleQuestions nearly solved: A new upperbound and baseline approach. In Proceedings of the 2018 Conference on Empirical Methods in Natural Language
Processing, pages 554-558, Brussels, Belgium. Association for Computational Linguistics.

Apoorv Saxena, Aditay Tripathi, and Partha Talukdar. 2020. Improving multi-hop question answering over knowledge graphs using knowledge base embeddings. In Proceedings of the 58th Annual Meeting of the Association for Computational Linguistics, pages 4498-4507, Online. Association for Computational Linguistics.

Kuldeep Singh, Ioanna Lytra, Arun Sethupat Radhakrishna, Saeedeh Shekarpour, Maria-Esther Vidal, and Jens Lehmann. 2020. No one is perfect: Analysing the performance of question answering components over the dbpedia knowledge graph. Journal of Web Semantics, 65:100594.

Daniil Sorokin and Iryna Gurevych. 2018. Mixing context granularities for improved entity linking on question answering data across entity categories. In Proceedings of the Seventh Joint Conference on Lexical and Computational Semantics, pages 65-75, New Orleans, Louisiana. Association for Computational Linguistics.

Haitian Sun, Andrew O Arnold, Tania Bedrax-Weiss, Fernando Pereira, and William W Cohen. 2020. Faithful embeddings for knowledge base queries. Advances in Neural Information Processing Systems, 33.

Haitian Sun, Tania Bedrax-Weiss, and William Cohen. 2019. PullNet: Open domain question answering with iterative retrieval on knowledge bases and text. In Proceedings of the 2019 Conference on Empirical Methods in Natural Language Processing and the 9th International Joint Conference on Natural Language Processing (EMNLP-IJCNLP), pages 23802390, Hong Kong, China. Association for Computational Linguistics.

Chuanqi Tan, Furu Wei, Pengjie Ren, Weifeng Lv, and Ming Zhou. 2017. Entity linking for queries by searching Wikipedia sentences. In Proceedings of the 2017 Conference on Empirical Methods in Natural Language Processing, pages 68-77, Copenhagen, Denmark. Association for Computational Linguistics.

Peng Wu, Shujian Huang, Rongxiang Weng, Zaixiang Zheng, Jianbing Zhang, Xiaohui Yan, and Jiajun Chen. 2019. Learning representation mapping for relation detection in knowledge base question answering. In Proceedings of the 57th Annual Meeting of the Association for Computational Linguistics, pages 6130-6139, Florence, Italy. Association for Computational Linguistics.

Wen-tau Yih, Matthew Richardson, Chris Meek, MingWei Chang, and Jina Suh. 2016. The value of semantic parse labeling for knowledge base question answering. In Proceedings of the 54th Annual Meeting of the Association for Computational Linguistics (Volume 2: Short Papers), pages 201-206, Berlin, 
Germany. Association for Computational Linguistics.

\section{A Model Hyperparameters}

We train Rigel models using the hyperparameters below on a single GPU machine with 16GB GPU memory (AWS p3.2xlarge). WebQSP requires more than 1 hop for question answering, leading to a larger knowledge graph, so we use a smaller batch size to avoid out of memory issues. Training with early stopping completes in approximately 4-7 hours depending on the model configuration used (Rigel-baseline, Rigel-ER, Rigel-E2E).

\begin{tabular}{lrr}
\hline Hyperparameter & SIQ & WQSP \\
\hline Batch Size & 32 & 6 \\
Gradient Accumulation & 8 & 32 \\
Max Training Steps & 20000 & 30000 \\
Learning Rate & $1 \mathrm{e}-4$ & $1 \mathrm{e}-4$ \\
Max Number of Hops & 1 & 3 \\
\hline
\end{tabular}

Table 2: Hyperparameters for training on WebQuestionsSP (WQSP) and SimpleQuestions (SIQ)

\section{B Examples}

The table below shows outputs of Rigel-E2E model on two questions from SimpleQuestions. In the first example, the model assigns high likelihood to the correct span and candidate entity. The inference module also assigns a high likelihood to the right relation (instance of), which leads to the correct answer entity.

In the second question, the model assigns higher likelihood to the sam edwards span, but it's not very confident and other spans such as sam and edwards receive similar scores. In addition, there's a large overlap between candidate entities of these spans (i.e. all produce candidates which are human and have place of birth property). This ambiguity in context leads to the ground truth question entity receiving a low likelihood. Even though the right relation is predicted by the inference module, the final answer entity is different from the answer label. 
Question: what is the category of the celestial object 1241 dysona?

Question Entity: Q137259 (1241 Dysona)

Answer Entity Q3863 (asteroid)

Span Likelihoods:

('1241 dysona', 0.965), ('celestial', 0.013), ('celestial object', 0.011),

('object', 0.009), ('1241', 0.002), ('the category', 0.0), ('what is', 0.0), ('category', 0.0)

Candidate Entity Likelihoods:

('Q137259', 0.998), ('Q6999', 0.0), ('Q66311333', 0.0), ('Q488383', 0.0), ․

Top Prediction:

('1241 dysona', 0.965) $\rightarrow$ (instance of, 1.000) $\rightarrow$ ('Q3863', 0.998)

Question: what is the place of birth of sam edwards?

Question Entity: Q472382 (Sam Edwards, Welsh Physicist)

Answer Entity Q23051 (Swansea)

Span Likelihoods:

('edwards', 0.366), ('sam edwards', 0.332), ('sam', 0.301), ('what is', 0.0), ('the place', 0.0),

('place', 0.0), ('the place of birth', 0.0), ('place of birth', 0.0), ('birth', 0.0)

Candidate Entity Likelihoods:

('Q3470479', 0.25), ('Q835638', 0.111), ('Q911493', 0.058), ('Q2691159', 0.017),

('Q20812281', 0.016), ('Q1816301', 0.014), ('Q47465190', 0.013), ('Q1118055', 0.011),

('Q58317511', 0.01), ('Q472382', 0.01), ('Q27925002', 0.01), ('Q52852726', 0.009), ‥

Top Prediction:

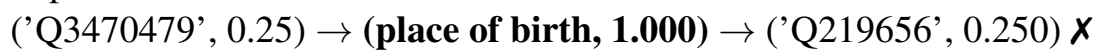

Table 3: Example outputs of Rigel-E2E on SimpleQuestions 